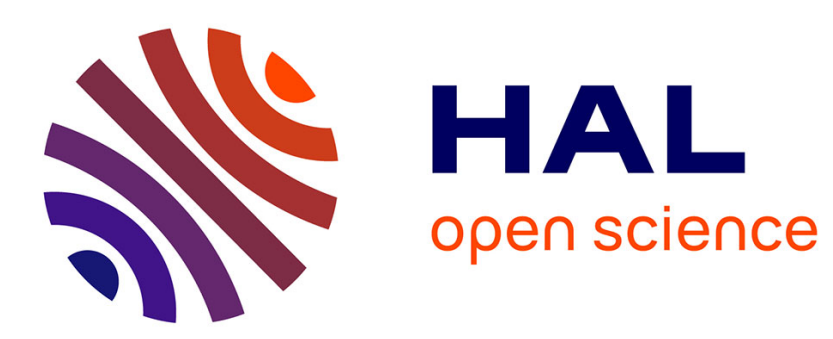

\title{
Strategic Communication with Decoder Side Information
}

Mael Le Treust, Tristan Tomala

\section{To cite this version:}

Mael Le Treust, Tristan Tomala. Strategic Communication with Decoder Side Information. IEEE ISIT 2021, Jul 2021, Melbourne (virtual), Australia. hal-03218977

\section{HAL Id: hal-03218977 \\ https://hal.science/hal-03218977}

Submitted on 6 May 2021

HAL is a multi-disciplinary open access archive for the deposit and dissemination of scientific research documents, whether they are published or not. The documents may come from teaching and research institutions in France or abroad, or from public or private research centers.
L'archive ouverte pluridisciplinaire HAL, est destinée au dépôt et à la diffusion de documents scientifiques de niveau recherche, publiés ou non, émanant des établissements d'enseignement et de recherche français ou étrangers, des laboratoires publics ou privés. 


\section{Strategic Communication with Decoder Side Information}

\author{
Maël Le Treust \\ ETIS UMR 8051 \\ CY Cergy Paris Université, ENSEA, CNRS \\ 95014 Cergy-Pontoise CEDEX, FRANCE \\ Email: mael.le-treust@ensea.fr
}

\author{
Tristan Tomala \\ GREGHEC UMR 2959 \\ HEC Paris \\ 78351 Jouy-en-Josas CEDEX, FRANCE \\ Email: tomala@hec.fr
}

\begin{abstract}
The strategic communication problem consists of a joint source-channel coding problem in which the encoder and the decoder optimize two arbitrary distinct distortion functions. This problem lies on the bridge between Information Theory and Game Theory. As in the persuasion game of Kamenica and Gentzkow, we consider that the encoder commits to an encoding strategy, then the decoder selects the optimal output symbol based on its Bayesian posterior belief. The informational content of the source affects differently the two distinct distortion functions, therefore each symbol is encoded in a specific way. In this work, we consider that the decoder has side information. Accordingly, we reformulate the Bayesian update of the decoder posterior beliefs and the optimal information disclosure policy of the encoder. We provide four different expressions of the solution, in terms of the expected encoder distortion optimized under an information constraint, and it in terms of convex closures of auxiliary distortion functions. We compute the encoder optimal distortion for the doubly symmetric binary source example.

A full version of this paper is accessible at: https://arxiv.org/abs/1911.04950
\end{abstract}

\section{INTRODUCTION}

Communication between autonomous devices that have distinct objectives is under study. This problem, referred to as the strategic communication problem, is at the crossroads of different disciplines such as Control Theory [1], [2], Computer Science [3] and Information Theory [4], [7], [8], [9], [10], [11], [12], where it was introduced by Akyol et al. in [5], [6].

Three different formulations of the strategic communication problem are originally proposed in the Game Theory literature, see [13]. The cheap talk game of Crawford and Sobel [14] relies on the Nash equilibrium solution. In the mechanism design problem of Jackson and Sonnenschein [15] the receiver commits to a prescribed decoding strategy, as the leader of a Stackelberg game. The hypothesis of decoder commitment is also considered in the mismatched rate-distortion problem in [16], [17]. In the persuasion game of Kamenica and Gentzkow [18] it is the sender who commits to a strategy whereas the decoder computes its Bayesian posterior belief and selects the optimal output symbol. In [19], we characterize the impact of the channel noise in the solution to the persuasion problem.

In this article, we extend these previous results with encoder commitment by considering that the decoder has side information. More specifically, we formulate a joint source-channel coding problem with decoder side information in which the encoder and the decoder are endowed with distinct distortion functions. Given an encoder strategy, the decoder selects an optimal strategy for its distortion function. The encoder anticipates the mismatch of the distortion functions and commits to implement the encoding strategy that minimizes its distortion.

The technical novelty consists in controlling the distance of the posterior beliefs induced by Wyner-Ziv's coding to the target posterior beliefs. This demonstrates that the Wyner-Ziv's encoding reveals nothing but the exact amount of information needed to implement the optimal decoding strategy. Consequently at the optimum the decoder produces a sequence of outputs which is almost the same as the one generated by the Wyner-Ziv's coding [20], for a specific probability distribution.

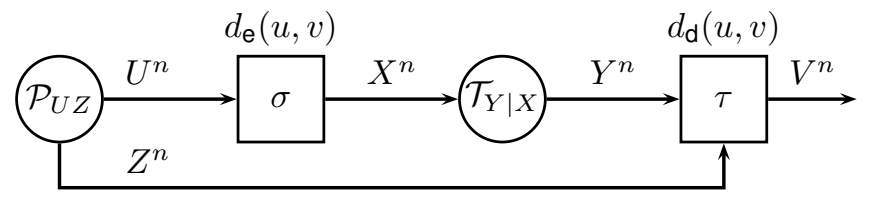

Fig. 1. The source $\mathcal{P}_{U Z}$ is i.i.d., the channel $\mathcal{T}_{Y \mid X}$ is memoryless. The encoder and the decoder have arbitrary mismatched distortion functions $d_{\mathrm{e}}(u, v) \neq d_{\mathrm{d}}(u, v)$.

\section{SYSTEM MODEL}

We denote by $\mathcal{U}, \mathcal{Z}, \mathcal{X}, \mathcal{Y}, \mathcal{V}$, the finite sets of information source, side information, channel inputs, channel outputs and decoder's outputs. Uppercase letters $U^{n}=\left(U_{1}, \ldots, U_{n}\right) \in$ $\mathcal{U}^{n}$ and $Z^{n}, X^{n}, Y^{n}, V^{n}$ stand for $n$-length sequences of random variables with $n \in \mathbb{N}^{\star}=\mathbb{N} \backslash\{0\}$, whereas lowercase letters $u^{n}=\left(u_{1}, \ldots, u_{n}\right) \in \mathcal{U}^{n}$ and $z^{n}, x^{n}, y^{n}, v^{n}$, stand for sequences of realizations. We denote by $\Delta(\mathcal{X})$ the set of probability distributions $\mathcal{Q}_{X}$ over $\mathcal{X}$. The support of $\mathcal{Q}_{X}$ is denoted by $\operatorname{supp} \mathcal{Q}_{X}=\{x \in \mathcal{X}, \mathcal{Q}(x)>0\}$.

We consider an i.i.d. information source and a memoryless channel distributed according to $\mathcal{P}_{U Z} \in \Delta(\mathcal{U} \times \mathcal{Z})$ and $\mathcal{T}_{Y \mid X}$ : $\mathcal{X} \rightarrow \Delta(\mathcal{Y})$, as depicted in Fig. 1 .

Definition 1 We define the encoding strategy by $\sigma: \mathcal{U}^{n} \longrightarrow$ $\Delta\left(\mathcal{X}^{n}\right)$ and the decoding strategy by $\tau: \mathcal{Y}^{n} \times \mathcal{Z}^{n} \longrightarrow \Delta\left(\mathcal{V}^{n}\right)$, and we denote by $\mathcal{P}^{\sigma, \tau}$ the distribution defined by

$$
\mathcal{P}^{\sigma, \tau}=\left(\prod_{t=1}^{n} \mathcal{P}_{U_{t} Z_{t}}\right) \sigma_{X^{n} \mid U^{n}}\left(\prod_{t=1}^{n} \mathcal{T}_{Y_{t} \mid X_{t}}\right) \tau_{V^{n} \mid Y^{n} Z^{n}}
$$

where $\sigma_{X^{n} \mid U^{n}}, \tau_{V^{n} \mid Y^{n} Z^{n}}$ denote the distributions of $\sigma, \tau$. 
Definition 2 The encoder and decoder distortion functions $d_{e}: \mathcal{U} \times \mathcal{V} \longrightarrow \mathbb{R}$ and $d_{d}: \mathcal{U} \times \mathcal{V} \longrightarrow \mathbb{R}$ are arbitrary and distinct, i.e. we consider any pair of functions. The long-run distortion functions $d_{e}^{n}(\sigma, \tau), d_{d}^{n}(\sigma, \tau)$ are given by

$$
d_{d}^{n}(\sigma, \tau)=\sum_{u^{n}, v^{n}} \mathcal{P}^{\sigma, \tau}\left(u^{n}, v^{n}\right) \cdot\left(\frac{1}{n} \sum_{t=1}^{n} d_{d}\left(u_{t}, v_{t}\right)\right) .
$$

Definition 3 Given $n \in \mathbb{N}^{\star}$, we define:

1. the set of decoder best responses to strategy $\sigma$ by

$$
B R_{d}(\sigma)=\underset{\tau}{\operatorname{argmin}} d_{d}^{n}(\sigma, \tau),
$$

2. the long-run encoder distortion value by

$$
D_{e}^{n}=\inf _{\sigma} \max _{\tau \in B R_{d}(\sigma)} d_{e}^{n}(\sigma, \tau) .
$$

In case $\mathrm{BR}_{\mathrm{d}}(\sigma)$ is not a singleton, we assume that the decoder selects the worst strategy for the encoder distortion $\max _{\tau \in \mathrm{BR}_{\mathrm{d}}(\sigma)} d_{\mathrm{e}}^{n}(\sigma, \tau)$, so that the solution is robust to the exact specification of the decoding strategy.

We aim at characterizing the asymptotic behavior of $D_{\mathrm{e}}^{n}$.

Definition 4 We consider an auxiliary random variable $W \in$ $\mathcal{W}$ with $|\mathcal{W}|=\min \left(|\mathcal{U}|+1,|\mathcal{V}|^{|\mathcal{Z}|}\right)$ and we define

$$
\mathbb{Q}=\left\{\mathcal{P}_{U Z} \mathcal{Q}_{W \mid U}, \max _{\mathcal{P}_{X}} I(X ; Y)-I(U ; W \mid Z) \geq 0\right\} .
$$

Given $\mathcal{Q}_{U Z W}$, we define the single-letter best responses

$$
\mathbb{A}_{d}\left(\mathcal{Q}_{U Z W}\right)=\underset{\mathcal{Q}_{V \mid Z W}}{\operatorname{argmin}} \mathbb{\mathbb { E } _ { \mathcal { Q } _ { V | Z W } }}\left[d_{d}(U, V)\right] .
$$

The encoder optimal distortion $D_{e}^{\star}$ is given by

$$
D_{e}^{\star}=\inf _{\mathcal{Q}_{U Z W} \in \mathbb{Q}} \max _{\substack{\left.\mathcal{Q}_{V \mid Z W} \in\right) \\ \mathbb{A}_{d}\left(\mathcal{Q}_{U Z W}\right)}} \mathbb{E}_{\substack{\mathcal{Q}_{V I Z W} \\ \mathcal{Q}_{V \mid Z W}}}\left[d_{e}(U, V)\right] .
$$

If $\mathbb{A}_{d}\left(\mathcal{Q}_{U Z W}\right)$ is not a singleton, the decoder selects the worst distribution $\mathcal{Q}_{V \mid Z W}$ from the encoder perspective.

\section{Theorem 1}

$$
\begin{aligned}
\forall n \in \mathbb{N}^{\star}, & D_{e}^{n} \geq D_{e}^{\star}, \\
\forall \varepsilon>0, \exists \bar{n} \in \mathbb{N}^{\star}, \forall n \geq \bar{n}, & D_{e}^{n} \leq D_{e}^{\star}+\varepsilon .
\end{aligned}
$$

The proof of Theorem 1 is stated in [22, App. B and C].

Sketch of proof of (7). For all $n \in \mathbb{N}^{\star}$, the converse result relies on the identification of the auxiliary random variables $W=\left(Y^{n}, Z^{T-1}, Z_{T+1}^{n}, T\right)$ and $(U, Z, V)=\left(U_{T}, Z_{T}, V_{T}\right)$, where $T$ is uniformly distributed over $\{1, \ldots, n\}$. We denote by $\mathcal{Q}_{U Z W}^{\sigma}, \mathcal{Q}_{V \mid Z W}^{\tau}$ the distributions induced by $(\sigma, \tau)$ over $(U, Z, W, V)$. In [21], it is proved that the Markov chain $Z-$ $U \bullet W$ holds and that $I(U ; W \mid Z) \leq \max _{\mathcal{P}_{X}} I(X ; Y)$, hence $\mathcal{Q}_{U Z W}^{\sigma} \in \mathbb{Q}$. We show that $d_{\mathrm{e}}^{n}(\sigma, \tau)=\mathbb{E}_{\substack{\mathcal{Q}_{U Z W}^{\sigma} \\ \mathcal{Q}_{V \mid Z W}^{U}}}\left[d_{\mathrm{e}}(U, V)\right]$ and $\left\{\mathcal{P}_{V \mid Z W}, \quad \exists \tau \in \mathrm{BR}_{\mathrm{d}}(\sigma), \quad \mathcal{Q}_{V \mid Z W}^{\tau}=\mathcal{P}_{V \mid Z W}\right\}=$ $\mathbb{A}_{d}\left(\mathcal{Q}_{U Z W}^{\sigma}\right)$. Then for any $\tilde{\sigma}$, we have

$$
\begin{aligned}
& \max _{\tau \in \mathrm{BR}_{\mathrm{d}}(\tilde{\sigma})} d_{\mathrm{e}}^{n}(\tilde{\sigma}, \tau)=\max _{\substack{\mathcal{P}_{V \mid Z W}, \exists \tau \in \mathrm{BR}_{\mathrm{d}}(\tilde{\sigma}) \\
\mathcal{Q}_{V \mid Z W}^{\tau}=\mathcal{P}_{V \mid Z W}}} \mathbb{E}_{\substack{\mathcal{Q}_{U Z W}^{\tilde{\sigma}} \\
\mathcal{P}_{V \mid Z W}}}\left[d_{\mathrm{e}}(U, V)\right] \\
& =\max _{\substack{\mathcal{Q}_{V \mid Z W} \in \\
\mathbb{A}_{\mathrm{d}}\left(\mathcal{Q}_{U Z W}^{\tilde{\sigma}}\right)}} \mathbb{E}_{\substack{\mathcal{Q}_{U Z W W}^{\tilde{\sigma}} \\
\mathcal{Q}_{V \mid Z W}}}\left[d_{\mathrm{e}}(U, V)\right] \\
& \inf _{\mathcal{Q}_{U Z W} \in \mathbb{Q}} \max _{\substack{\mathcal{Q}_{V \mid Z W} \in \\
\mathbb{A}_{\mathrm{d}}\left(\mathcal{Q}_{U Z W}\right)}} \mathbb{E}_{\substack{\mathcal{Q}_{U Z W} \\
\mathcal{Q}_{V \mid Z W}}}\left[d_{\mathrm{e}}(U, V)\right]=D_{\mathrm{e}}^{\star},
\end{aligned}
$$

which implies (7)

Note that the sequence $\left(n D_{\mathrm{e}}^{n}\right)_{n \in \mathbb{N}^{\star}}$ is sub-additive. Indeed, when $\sigma$ is the concatenation of several encoding strategies, the optimal $\tau$ in (3) is the concatenation of the optimal decoding strategies. Theorem 1 and Fekete's lemma, show that

$$
D_{\mathrm{e}}^{\star}=\lim _{n \rightarrow+\infty} D_{\mathrm{e}}^{n}=\inf _{n \in \mathbb{N}^{\star}} D_{\mathrm{e}}^{n} .
$$

\section{Convex Closure Formulation}

We denote by vex $f: \mathcal{X} \rightarrow \mathbb{R} \cup\{-\infty\}$ the convex closure of a function $f$, i.e. the largest convex function everywhere smaller than $f$ on $X$. We reformulate $D_{\mathrm{e}}^{\star}$ in terms of a convex closure, similarly to $[18$, Corollary 1$]$.

Lemma 1 For all $\mathcal{Q}_{W \mid U} \in \Delta(\mathcal{W})^{|\mathcal{U}|}$, for all $(u, z, w) \in$ $\operatorname{supp}\left(\mathcal{P}_{U Z} \mathcal{Q}_{W \mid U}\right)$ we have

$$
\mathcal{Q}(u \mid w, z)=\frac{\mathcal{Q}(u \mid w) \mathcal{P}(z \mid u)}{\sum_{u^{\prime}} \mathcal{Q}\left(u^{\prime} \mid w\right) \mathcal{P}\left(z \mid u^{\prime}\right)} .
$$

The proof is direct. The conditional distribution $\mathcal{Q}_{U \mid W Z} \in$ $\Delta(\mathcal{U})^{|\mathcal{W} \times \mathcal{Z}|}$ reformulates in terms of $\mathcal{Q}_{U \mid W} \in \Delta(\mathcal{U})^{|\mathcal{W}|}$.

Definition 5 For $p \in \Delta(\mathcal{U})$, the decoder selects $v^{\star}(p)$ in

$$
\mathcal{V}^{\star}(p)=\underset{v \in \operatorname{argmin} \mathbb{E}_{p}\left[d_{d}(U, v)\right]}{\operatorname{argmax}} \mathbb{E}_{p}\left[d_{e}(U, v)\right],
$$

and the robust distortion function writes

$$
\psi_{e}(p)=\mathbb{E}_{p}\left[d_{e}\left(U, v^{\star}(p)\right)\right], \quad v^{\star}(p) \in \mathcal{V}^{\star}(p) .
$$

Definition 6 For $p \in \Delta(\mathcal{U})$, the average distortion function $\Psi_{e}(p)$ and the average entropy function $h(p)$ are defined by

$$
\begin{gathered}
\Psi_{e}(p)=\sum_{u, z} p(u) \mathcal{P}(z \mid u) \cdot \psi_{e}\left(\frac{p(\cdot) \mathcal{P}(z \mid \cdot)}{\sum_{u^{\prime}} p\left(u^{\prime}\right) \mathcal{P}\left(z \mid u^{\prime}\right)}\right) \\
h(p)=\sum_{u, z} \mathcal{P}(z) \frac{p(u) \mathcal{P}(z \mid u)}{\sum_{u^{\prime}} p\left(u^{\prime}\right) \mathcal{P}\left(z \mid u^{\prime}\right)} \\
\quad \times \log _{2} \frac{\sum_{u^{\prime}} p\left(u^{\prime}\right) \mathcal{P}\left(z \mid u^{\prime}\right)}{p(u) \mathcal{P}(z \mid u)} .
\end{gathered}
$$

The function $h(p)$ is the conditional entropy $H(U \mid Z)$ evaluated with respect to $p \cdot \mathcal{P}_{Z \mid U}$ instead of $\mathcal{P}_{U Z}$.

Lemma 2 The function $h(p)$ is concave in $p \in \Delta(\mathcal{U})$. 
Proof. [Lemma 2] The entropy $H(U)$ is concave in $p \in \Delta(\mathcal{U})$, the mutual information $I(U ; Z)$ is convex in $p \in \Delta(\mathcal{U})$ for fixed $\mathcal{P}_{Z \mid U}$ and moreover $H(U \mid Z)=H(U)-I(U ; Z)$.

\section{Theorem 2}

$$
\begin{aligned}
D_{e}^{\star}= & \inf \left\{\sum_{w \in \mathcal{W}} \lambda_{w} \Psi_{e}\left(p_{w}\right), \quad \sum_{w \in \mathcal{W}} \lambda_{w} p_{w}=\mathcal{P}_{U},\right. \\
& \left.\sum_{w \in \mathcal{W}} \lambda_{w} h\left(p_{w}\right) \geq H(U \mid Z)-\max _{\mathcal{P}_{X}} I(X ; Y)\right\},
\end{aligned}
$$

where the infimum is taken over $\left(\lambda_{w}, p_{w}\right)_{w \in \mathcal{W}}$ with $|\mathcal{W}|=$ $\min \left(|\mathcal{U}|+1,|\mathcal{V}|^{|\mathcal{Z}|}\right)$, such that for each $w \in \mathcal{W}, p_{w} \in \Delta(\mathcal{U})$, $\lambda_{w} \in[0,1]$, and $\sum_{w \in \mathcal{W}} \lambda_{w}=1$.

The proof of Theorem 2 is stated in [22, App. A]. It is a consequence of the Markov chain property $Z \bullet U \bullet W$. Note that all the channels such that $\max _{\mathcal{P}_{X}} I(X ; Y) \geq H(U \mid Z)$ lead to the same value for $D_{\mathrm{e}}^{\star}$. The optimal parameters $\left(\lambda_{w}^{\star}, p_{w}^{\star}\right)_{w \in \mathcal{W}}$ in (18) are referred to as the optimal splitting of the prior distribution $\mathcal{P}_{U}$, see [24]. When removing the decoder side information, e.g. $|\mathcal{Z}|=1$, and changing the infimum into a supremum, we recover the value of the optimal splitting problem of [19, Definition 2.4].

- Since $\sum_{w} \lambda_{w} h\left(p_{w}\right)=H(U \mid Z, W)$, the information constraint in (18) is a reformulation of $I(U ; W \mid Z) \leq$ $\max _{\mathcal{P}_{X}} I(X ; Y)$.

- The dimension of the problem (18) is $|\mathcal{U}|+1$. Caratheodory's Lemma [23, Corollary 17.1.5, pp. 157] induces the cardinality bound $|\mathcal{W}| \leq|\mathcal{U}|+1$.

- The cardinality of $\mathcal{W}$ is also restricted by the vector of recommended symbols $|\mathcal{W}| \leq|\mathcal{V}|^{|\mathcal{Z}|}$, telling to the decoder which symbol $v \in \mathcal{V}$ to select when the side information is $z \in \mathcal{Z}$.

The encoder optimal distortion $D_{\mathrm{e}}^{\star}$ can be reformulated in terms of Lagrangian and in terms of the convex closure of

$$
\tilde{\Psi}_{\mathrm{e}}(p, \nu)=\left\{\begin{array}{l}
\Psi_{\mathrm{e}}(p), \text { if } \nu \leq h(p) \\
+\infty, \text { otherwise }
\end{array}\right.
$$

\section{Theorem 3}

$$
\begin{aligned}
D_{e}^{\star}=\sup _{t \geq 0}\left\{\operatorname{vex}\left[\Psi_{e}+t \cdot h\right]\left(\mathcal{P}_{U}\right)\right. \\
\left.-t \cdot\left(H(U \mid Z)-\max _{\mathcal{P}_{X}} I(X ; Y)\right)\right\} \\
=\operatorname{vex} \tilde{\Psi}_{e}\left(\mathcal{P}_{U}, H(U \mid Z)-\max _{\mathcal{P}_{X}} I(X ; Y)\right) .
\end{aligned}
$$

Equation (20) is the convex closure of a Lagrangian with the information constraint. Equation (21) corresponds to the convex closure of a a bi-variate function where the information constraint requires an additional dimension. The proof follows directly from [19, Theorem 3.3, pp. 37] by replacing concave closure by convex closure.
Remark 1 When $d_{e}=d_{d}$, then $\psi_{e}(p)=\min _{v} \mathbb{E}_{p}\left[d_{e}(U, v)\right]$ and we obtain a reformulation of the Wyner-Ziv's solution [20]

$$
\begin{aligned}
D_{e}^{\star}= & \inf _{\substack{\left(\lambda_{w}, p_{w}\right)_{w \in \mathcal{W}}, \sum_{w} \lambda_{w}=1}}\left\{\sum_{w \in \mathcal{W}} \lambda_{w} \sum_{u, z} p_{w}(u) \mathcal{P}(z \mid u)\right. \\
& \times \min _{v} \mathbb{E} \frac{p_{w}(\cdot) \mathcal{P}(z \mid \cdot)}{\sum_{u^{\prime} p_{w}\left(u^{\prime}\right) \mathcal{P}\left(z \mid u^{\prime}\right)}}\left[d_{e}(U, v)\right], \quad \sum_{w \in \mathcal{W}} \lambda_{w} p_{w}=\mathcal{P}_{U} \\
& \left.\sum_{w \in \mathcal{W}} \lambda_{w} h\left(p_{w}\right) \geq H(U \mid Z)-\max _{\mathcal{P}_{X}} I(X ; Y)\right\}
\end{aligned}
$$

When $d_{e}=-d_{d}$, then $\mathcal{V}^{\star}(p)=\operatorname{argmax}_{v} \mathbb{E}_{p}\left[d_{e}(U, v)\right]$ and both functions $\psi_{e}(p), \Psi_{e}(p)$ are convex in $p \in \Delta(\mathcal{U})$. By Jensen's inequality, the infimum in (18) is achieved by $p_{w}^{\star}=$ $\mathcal{P}_{U}, \forall w \in \mathcal{W}$, i.e. no information is transmitted and $D_{e}^{\star}=$ $\max _{v} \mathbb{E}_{\mathcal{P}_{U}}\left[d_{e}(U, v)\right]$.

\section{Doubly Symmetric Binary Source}

We consider the doubly symmetric binary source (DSBS) example introduced by Wyner-Ziv in [20, Sec. II], depicted in Fig. 2 with parameters $\left(p_{0}, \delta_{0}, \delta_{1}\right) \in[0,1]^{3}$. The cardinality bound is $|\mathcal{W}|=\min \left(|\mathcal{U}|+1,|\mathcal{V}|^{|\mathcal{Z}|}\right)=3$, hence the random variable $W$ is drawn according to the conditional probability distribution $\mathcal{Q}_{W \mid U}$ with parameters $\left(\alpha_{k}, \beta_{k}\right)_{k \in\{1,2,3\}} \in[0,1]^{6}$ such that $\sum_{k} \alpha_{k}=\sum_{k} \beta_{k}=1$.

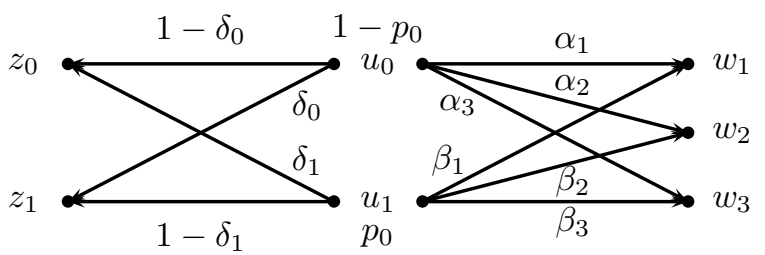

Fig. 2. Joint probability distribution $\mathcal{P}(u, z) \mathcal{Q}(w \mid u)$ with $|\mathcal{W}|=3$ depending on parameters $p_{0}, \delta_{0}, \delta_{1},\left(\alpha_{k}, \beta_{k}\right)_{k \in\{1,2,3\}}$ that belong to $[0,1]$.

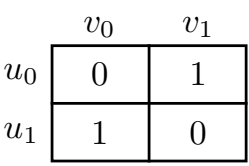

\begin{tabular}{c|c|c|} 
& \multicolumn{1}{c}{$v_{0}$} & \multicolumn{1}{c}{$v_{1}$} \\
\cline { 2 - 3 }$u_{0}$ & 0 & $1+\kappa$ \\
\cline { 2 - 3 }$u_{1}$ & 1 & $\kappa$ \\
\cline { 2 - 3 } & &
\end{tabular}

Fig. 3. Encoder distortion $d_{\mathrm{e}}(u, v)$. Fig. 4. Decoder distortion $d_{\mathrm{d}}(u, v)$.

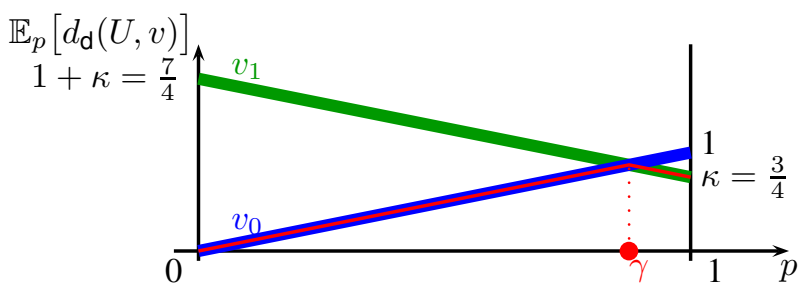

Fig. 5. Decoder's expected distortion $\mathbb{E}_{p}\left[d_{\mathrm{d}}(U, v)\right]=(1-p) \cdot d_{\mathrm{d}}\left(u_{0}, v\right)+$ $p \cdot d_{\mathrm{d}}\left(u_{1}, v\right)$ for $v \in\left\{v_{0}, v_{1}\right\}$ depending on the belief $\mathcal{Q}\left(u_{1} \mid w, z\right) \in[0,1]$.

The distortion functions are given by Fig. 3 and 4 for which the extra cost $\kappa \in[0,1]$ in the decoder distortion may capture a computing cost, an energy cost, or the fact that an estimation error of the symbol $v_{1}$ is more harmful than an estimation error of the symbol $v_{0}$. 


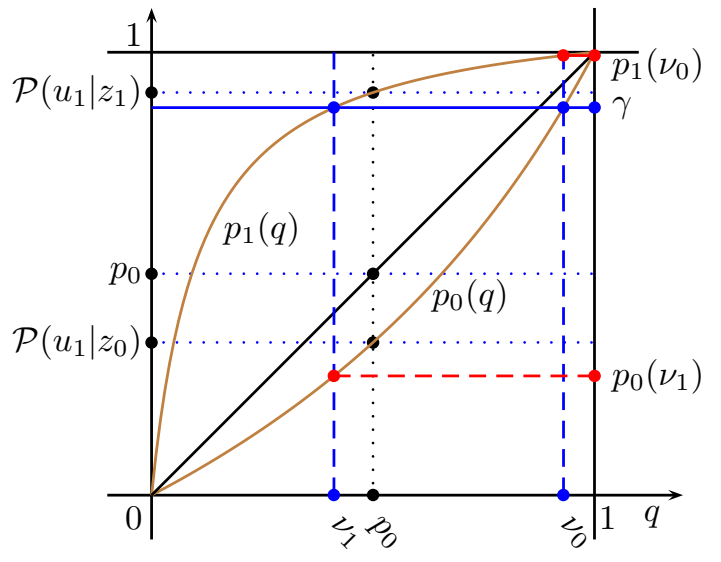

Fig. 6. Equations (23) and (24), depending on the interim belief $q \in[0,1]$, for $p_{0}=0.5, \delta_{1}=0.05, \delta_{2}=0.5$ and $\gamma=0.875$.

The optimal decision for the decoder depends on the posterior belief $\mathcal{Q}_{U}(\cdot \mid w, z) \in \Delta(\mathcal{U})$ after observing the symbols $(w, z)$. We denote by $\gamma=\frac{1+\kappa}{2}=\frac{7}{8}$ the belief threshold at which the decoder changes from symbol $v_{0}$ to $v_{1}$, as in Fig. 5. The decoder chooses $v_{0}^{\star}$ (resp. $v_{1}^{\star}$ ) when the posterior belief belongs to $[0, \gamma]$ (resp. $] \gamma, 1]$ ).

The correlation of $(U, Z)$ is fixed whereas the correlation of $(U, W)$ is selected by the encoder. Lemma 1 formulates the posterior belief $\mathcal{Q}_{U \mid W Z}$ in terms of the iterim belief $\mathcal{Q}_{U \mid W}$. For the symbols $w \in \mathcal{W}, z_{0} \in \mathcal{Z}, z_{1} \in \mathcal{Z}$ we have

$$
\begin{aligned}
& \mathcal{Q}\left(u_{1} \mid w, z_{0}\right)=\frac{q \delta_{1}}{(1-q)\left(1-\delta_{0}\right)+q \delta_{1}}=: p_{0}(q), \\
& \mathcal{Q}\left(u_{1} \mid w, z_{1}\right)=\frac{q\left(1-\delta_{1}\right)}{(1-q) \delta_{0}+q\left(1-\delta_{1}\right)}=: p_{1}(q) .
\end{aligned}
$$

Equations (23) and (24) are depicted on Fig. 6. Given the belief threshold $\gamma=\frac{7}{8}$, we define $\nu_{0}$ and $\nu_{1}$ such that

$$
\begin{aligned}
& \gamma=p_{0}\left(\nu_{0}\right) \Longleftrightarrow \nu_{0}=\frac{\gamma\left(1-\delta_{0}\right)}{\delta_{1}(1-\gamma)+\gamma\left(1-\delta_{0}\right)}, \\
& \gamma=p_{1}\left(\nu_{1}\right) \Longleftrightarrow \nu_{1}=\frac{\gamma \delta_{0}}{\gamma \delta_{0}+\left(1-\delta_{1}\right)(1-\gamma)} .
\end{aligned}
$$

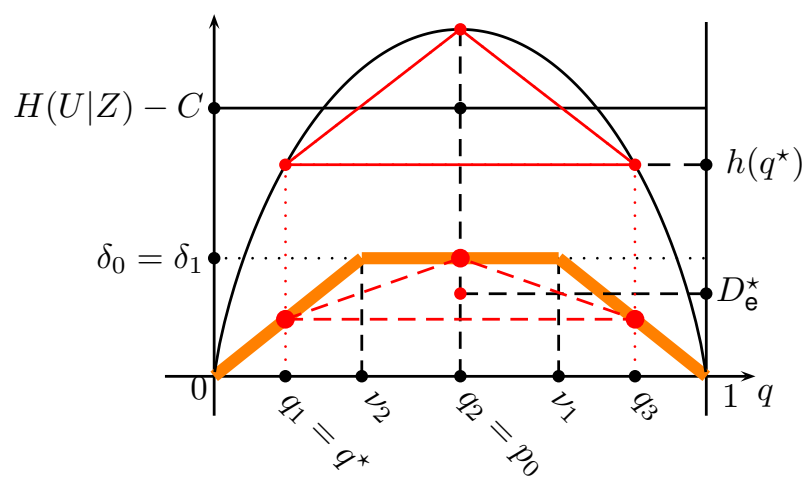

Fig. 7. The optimal splitting has three posteriors when $C \in[0, H(U \mid Z)-$ $\left.h\left(q^{\star}\right)\right]$, with $p_{0}=0.5, \delta_{0}=\delta_{1}=0.3, C=0.2, \kappa=0$, then $D_{\mathrm{e}}^{\star}=0.2098$.
Without loss of generality, we assume that $\delta_{0}+\delta_{1}<1 \Longleftrightarrow$ $\nu_{1}<\nu_{0}$. The robust and average distortion functions writes

$$
\begin{aligned}
\psi_{\mathrm{e}}(p)= & p \cdot \mathbb{1}(p \leq \gamma)+(1-p) \cdot \mathbb{1}(p>\gamma), \\
\Psi_{\mathrm{e}}(q)= & \operatorname{Pr}_{q}\left(z_{0}\right) \cdot \psi_{\mathrm{e}}\left(p_{0}(q)\right)+\operatorname{Pr}_{q}\left(z_{1}\right) \cdot \psi_{\mathrm{e}}\left(p_{1}(q)\right) \\
= & q \cdot \mathbb{1}\left(q \leq \nu_{1}\right)+(1-q) \cdot \mathbb{1}\left(q>\nu_{0}\right) \\
& +\left(q \delta_{1}+(1-q) \delta_{0}\right) \cdot \mathbb{1}\left(\nu_{1}<q \leq \nu_{0}\right) .
\end{aligned}
$$

The average distortion function $\Psi_{\mathrm{e}}(q)$ is depicted by the orange lines in Fig. 7, Fig. 8 and Fig. 11, where the black curve is the average entropy $h(q)=H_{b}(q)+(1-q) \cdot H_{b}\left(\delta_{0}\right)+$ $q \cdot H_{b}\left(\delta_{1}\right)-H_{b}\left((1-q) \delta_{0}+q\left(1-\delta_{1}\right)\right)$ and $H_{b}$ denotes the binary entropy.

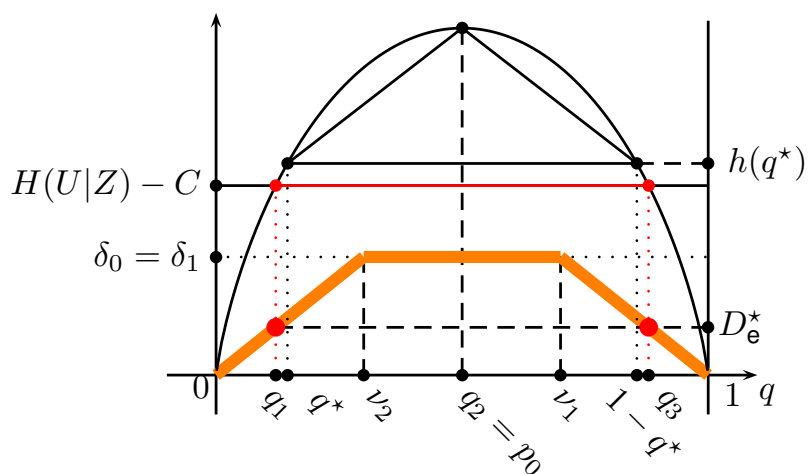

Fig. 8. The optimal splitting has only two posteriors when $C \in[H(U \mid Z)-$ $\left.h\left(q^{\star}\right), H(U \mid Z)\right]$, with $p_{0}=0.5, \delta_{0}=\delta_{1}=0.3, C=0.4, \kappa=0$, then $D_{\mathrm{e}}^{\star}=0.1212$.

The optimal splitting has posteriors $\left(q_{1}, q_{2}, q_{3}\right) \in[0,1]^{3}$ with respective weights $\left(\lambda_{1}, \lambda_{2}, \lambda_{3}\right) \in[0,1]^{3}$ that satisfy

$$
\begin{aligned}
1 & =\lambda_{1}+\lambda_{2}+\lambda_{3}, \\
p_{0} & =\lambda_{1} q_{1}+\lambda_{2} q_{2}+\lambda_{3} q_{3}, \\
H(U \mid Z)-C & =\lambda_{1} \cdot h\left(q_{1}\right)+\lambda_{2} \cdot h\left(q_{2}\right)+\lambda_{3} \cdot h\left(q_{3}\right) .
\end{aligned}
$$

Equation (32) is satisfied when the information constraint is binding, therefore we obtain [19, Equations (58) - (60)]. Without loss of generality, we assume that $q_{1} \in\left[0, \nu_{1}\left[, q_{2} \in\right.\right.$ $\left[\nu_{1}, \nu_{2}\left[, q_{3} \in\left[\nu_{2}, 1\right]\right.\right.$ and characterize the optimal solution in three different scenarios. According to the Splitting Lemma [24], we have for $k \in\{, 1,2,3\}$,

$$
\begin{aligned}
& \mathcal{Q}\left(w_{k} \mid u_{0}\right)=\mathcal{Q}\left(w_{k}\right) \frac{1-\mathcal{Q}\left(u_{1} \mid w_{k}\right)}{1-\mathcal{P}\left(u_{1}\right)}=\lambda_{k} \frac{1-q_{k}}{1-p_{0}}=\alpha_{k}, \\
& \mathcal{Q}\left(w_{k} \mid u_{1}\right)=\mathcal{Q}\left(w_{k}\right) \frac{\mathcal{Q}\left(u_{1} \mid w_{k}\right)}{\mathcal{P}\left(u_{1}\right)}=\lambda_{k} \frac{q_{k}}{p_{0}}=\beta_{k} .
\end{aligned}
$$

\section{A. Wyner-Ziv's Example With Equal Distortions}

We consider $p_{0}=0.5, \delta_{0}=\delta_{1}=0.3, \kappa=0$, hence both encoder and decoder minimize the Hamming distortion and hence $\gamma=\frac{1}{2}$. The average distortion and average entropy write

$$
\begin{aligned}
\Psi_{\mathrm{e}}(q)= & q \cdot \mathbb{1}(q \leq \delta)+\delta \cdot \mathbb{1}(\delta<q \leq 1-\delta) \\
& +(1-q) \cdot \mathbb{1}(q>1-\delta), \\
h(q)= & H(U \mid Z)+H_{b}(q)-H_{b}(q \star \delta),
\end{aligned}
$$


with the notation $q \star \delta=(1-q) \delta+q(1-\delta)$.

Proposition 1 We denote by $q^{\star}$ the unique solution to

$$
h^{\prime}(q)=\frac{H(U \mid Z)-h(q)}{\delta-q} .
$$

1) If $C \in\left[0, H(U \mid Z)-h\left(q^{\star}\right)\right]$ then at the optimum (Fig. 7)

$$
\begin{aligned}
& q_{1}=q^{\star}=1-q_{3}, \quad q_{2}=\frac{1}{2}, \\
& \lambda_{1}=\frac{1}{2} \cdot \frac{C}{H(U \mid Z)-h\left(q^{\star}\right)}=\lambda_{3}=\frac{1-\lambda_{2}}{2},
\end{aligned}
$$

which correspond to the distribution parameters $\alpha_{1}=(1-$ $\left.q^{\star}\right) \cdot \frac{C}{H(U \mid Z)-h\left(q^{\star}\right)}=\beta_{3}, \alpha_{2}=1-\frac{C}{H(U \mid Z)-h\left(q^{\star}\right)}=\beta_{2}, \alpha_{3}=$ $q^{\star} \cdot \frac{C}{H(U \mid Z)-h\left(q^{\star}\right)}=\beta_{1}$, and to the optimal distortion

$$
D_{e}^{\star}=\delta-C \cdot \frac{\delta-q^{\star}}{H(U \mid Z)-h\left(q^{\star}\right)} .
$$

2) If $C \in\left[H(U \mid Z)-h\left(q^{\star}\right), H(U \mid Z)\right]$ then at the optimum (Fig. 8) $q_{1}=h^{-1}(H(U \mid Z)-C)=1-q_{3}, q_{2}=\frac{1}{2}, \lambda_{1}=$ $\frac{1}{2}=\lambda_{3}, \quad \lambda_{2}=0$, which correspond to the distribution parameters $\alpha_{1}=1-h^{-1}(H(U \mid Z)-C)=1-\alpha_{3}=\beta_{3}=$ $1-\beta_{1}$ and $\alpha_{2}=\beta_{2}=0$ and to the optimal distortion

$$
D_{e}^{\star}=h^{-1}(H(U \mid Z)-C)
$$

where the notation $h^{-1}(H(U \mid Z)-C)$ stands for the unique solution $q \in[0,1]$ of the equation $h(q)=H(U \mid Z)-C$.

3) If $C>H(U \mid Z)$, then the optimal splitting rely on the two extreme posterior beliefs $(0,1)$ and $D_{e}^{\star}=0$.

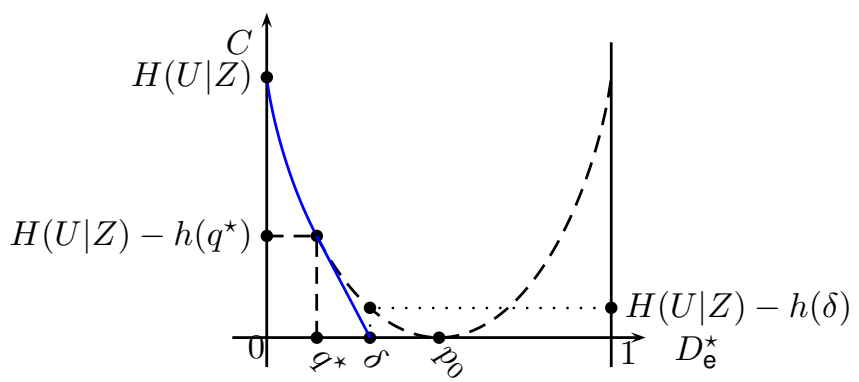

Fig. 9. Optimal trade-off between the capacity $C$ and the optimal distortion $D_{\mathrm{e}}^{\star}$ for the DSBS with parameters $p_{0}=0.5, \delta_{0}=\delta_{1}=0.3, \kappa=0$.

The proof of Proposition 1 is provided in [22, App. D]. When $C \leq H(U \mid Z)-h\left(q^{\star}\right)$, the optimal strategy consists of a time-sharing between $\left(D_{\mathrm{e}}^{\star}, C\right)=\left(q^{\star}, H(U \mid Z)-h\left(q^{\star}\right)\right)$ and the zero rate point $(\delta, 0)$, as depicted in Fig. 9.

\section{B. Mismatched Distortions Without Side Information}

We consider $p_{0}=0.5, C=0.2, \kappa=\frac{3}{4}$ and $\delta_{0}=\delta_{1}=0.5$ so that $Z$ is independent of $U$, as in [19]. We have $H_{b}\left(\delta_{0}\right)=$ $H_{b}\left(\delta_{1}\right)=H_{b}\left((1-q) \delta_{0}+q\left(1-\delta_{1}\right)\right)=1$ and $\nu_{1}=\nu_{2}=\gamma=\frac{7}{8}$. The average entropy and average distortion write

$$
\begin{aligned}
h(q) & =H_{b}(q), \\
\Psi_{\mathrm{e}}(q) & =\psi_{\mathrm{e}}(q)=p \cdot \mathbb{1}(p \leq \gamma)+(1-p) \cdot \mathbb{1}(p>\gamma),
\end{aligned}
$$

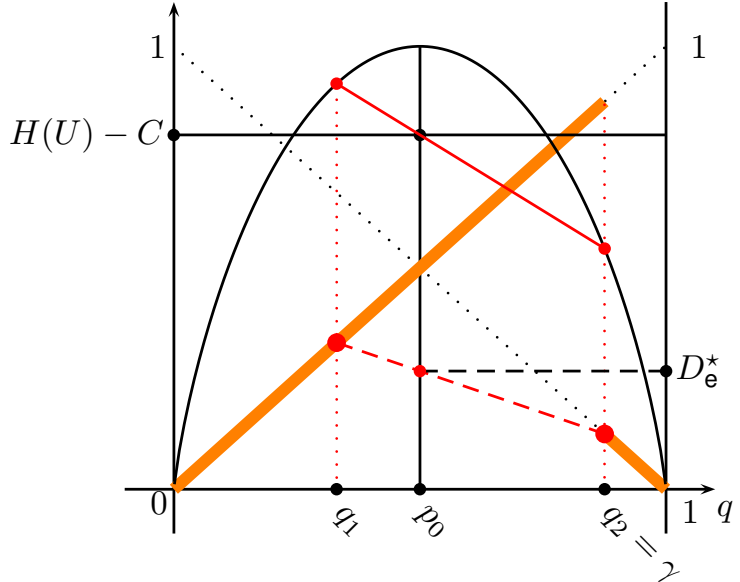

Fig. 10. For $p_{0}=0.5, \delta_{0}=\delta_{1}=0.5, C=0.2, \kappa=\frac{3}{4}, D_{\mathrm{e}}^{\star}=0.2668$.

and are depicted in Fig. 10. Applying [19, Corollary 3.5], the optimal splitting has two posteriors, i.e. $|\mathcal{W}|=2$, and satisfy

$$
\frac{p_{0}-q_{2}}{q_{1}-q_{2}} \cdot H_{b}\left(q_{1}\right)+\frac{q_{1}-p_{0}}{q_{1}-q_{2}} \cdot H_{b}\left(q_{2}\right) \geq H(U)-C .
$$

By numerical optimization, the above inequality is satisfied for $p_{0}=0.5, \delta_{0}=\delta_{1}=0.5, C=0.2, \kappa=\frac{3}{4}$, hence the optimal distortion is achieved by using $q_{2}=\gamma$, as in Fig. 10 .

\section{Mismatched Distortions With Side Information}

We consider $p_{0}=0.5, \delta_{0}=0.05, \delta_{1}=0.5, C=0.2$, $\kappa=\frac{3}{4}$. By numerical simulation, we determine the optimal triple of posteriors $\left(q_{1}, q_{2}, q_{3}\right)$ represented by the red dots in Fig. 11, corresponding to $D_{\mathrm{e}}^{\star}=0.1721$. The parameters of the optimal strategy in Fig. 2, are given by the following table.

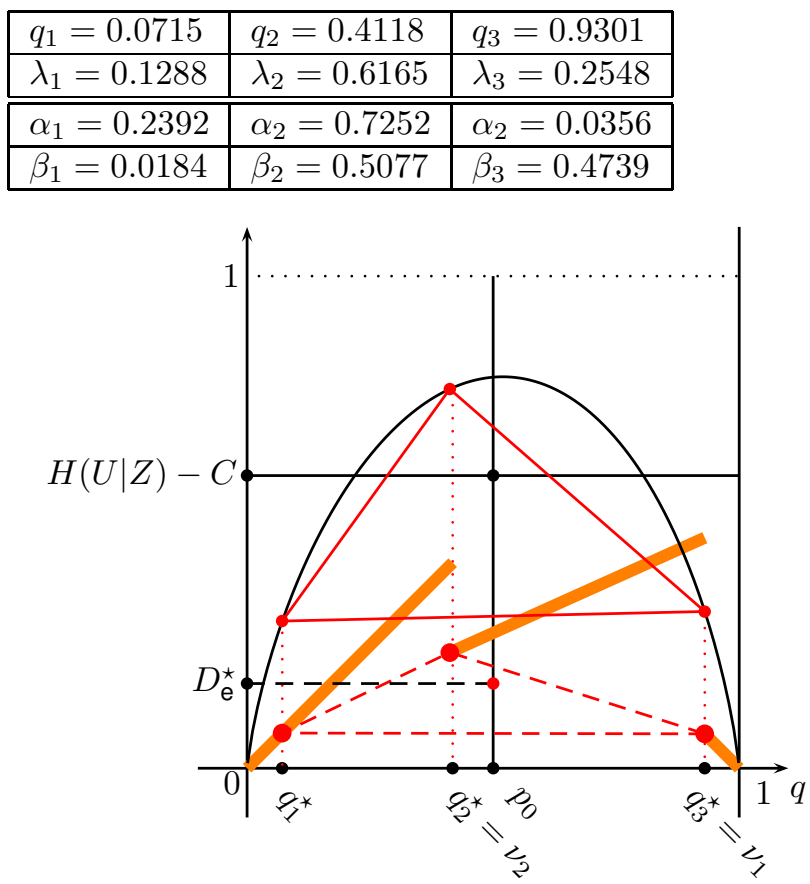

Fig. 11. $p_{0}=0.5, \delta_{0}=0.05, \delta_{1}=0.5, C=0.2, \kappa=\frac{3}{4}, D_{\mathrm{e}}^{\star}=0.1721$. 


\section{REFERENCES}

[1] S. Sarıtaş, S. Yüksel, and S. Gezici, "Quadratic multi-dimensional signaling games and affine equilibria," IEEE Transactions on Automatic Control, vol. 62, no. 2, pp. 605-619, Feb. 2017.

[2] S. Sarıtaş and S. Y "Dynamic signaling games with quadratic criteria under nash and stackelberg equilibria," Automatica, vol. 115, p. 108883, 2020.

[3] S. Dughmi, R. Niazadeh, A. Psomas, and S. M. Weinberg, "Persuasion and incentives through the lens of duality," in International Conference on Web and Internet Economics. Springer, 2019, pp. 142-155.

[4] M. Le Treust and T. Tomala, "Information design for strategic coordination of autonomous devices with non-aligned utilities," IEEE Proc. of the 54th Allerton conference, Monticello, Illinois, 2016, pp. 233-242.

[5] E. Akyol, C. Langbort, and T. Başar, "On the role of side information in strategic communication," 2016 IEEE International Symposium on Information Theory (ISIT), pp. 1626-1630.

[6] E. Akyol, C. Langbort, and T. Başar, "Information-theoretic approach to strategic communication as a hierarchical game," Proceedings of the IEEE, vol. 105, no. 2, pp. 205-218, 2017.

[7] M. Le Treust and T. Tomala, "Strategic coordination with state information at the decoder," Proc. of 2018 International Zurich Seminar on Information and Communication.

[8] M. Le Treust and T. Tomala, "Information-theoretic limits of strategic communication," [on-line] available: https://arxiv.org/abs/1807.05147, 2018.

[9] S. Sarıtaş, P. Furrer, S. Gezici, T. Linder, and S. Yüksel, "On the number of bins in equilibria for signaling games," in 2019 IEEE International Symposium on Information Theory (ISIT), pp. 972-976.

[10] A. S. Vora and A. A. Kulkarni, "Achievable rates for strategic communication," in 2020 IEEE International Symposium on Information Theory (ISIT), pp. 1379-1384.

[11] A. S. Vora and A. A. Kulkarni, "Information extraction from a strategic sender: The zero error case," [on-line] available: https://arxiv.org/abs/2006.10641, 2020.

[12] S. G. S. Y. Ertan Kazıklı, Serkan Sarıtaş, "Optimal signaling with mismatch in priors of an encoder and decoder," [on-line] available: https://arxiv.org/abs/2101.00799, Jan. 2021.

[13] M. Le Treust and T. Tomala, "Point-to-point strategic communication," in 2020 IEEE Information Theory Workshop.

[14] V. P. Crawford and J. Sobel, "Strategic information transmission," Econometrica, vol. 50, no. 6, pp. 1431-1451, 1982.

[15] M. O. Jackson and H. F. Sonnenschein, "Overcoming incentive constraints by linking decisions," Econometrica, vol. 75, no. 1, pp. 241 257, January 2007.

[16] A. Lapidoth, "On the role of mismatch in rate distortion theory," IEEE Transactions on Information Theory, vol. 43, no. 1, pp. 38-47, Jan. 1997.

[17] J. Scarlett, A. Guillen i Fàbregas, A. Somekh-Baruch, and A. Martinez, "Information-theoretic foundations of mismatched decoding," Foundations and Trends in Communications and Information Theory, vol. 17, no. 2-3, pp. 149-401, 2020.

[18] E. Kamenica and M. Gentzkow, "Bayesian persuasion," American Economic Review, vol. 101, pp. 2590-2615, 2011.

[19] M. Le Treust and T. Tomala, "Persuasion with limited communication capacity," Journal of Economic Theory, vol. 184, p. 104940, 2019.

[20] A. D. Wyner and J. Ziv, "The rate-distortion function for source coding with side information at the decoder," IEEE Transactions on Information Theory, vol. 22, no. 1, pp. 1-11, Jan. 1976.

[21] N. Merhav and S. Shamai, "On joint source-channel coding for the Wyner-Ziv source and the Gel'fand-Pinsker channel," IEEE Transactions on Information Theory, vol. 49, no. 11, pp. 2844-2855, 2003.

[22] M. Le Treust and T. Tomala, "Strategic communication with side information at the decoder," [on-line] available: https://arxiv.org/abs/1911.04950, Nov. 2019.

[23] R. Rockafellar, Convex Analysis, ser. Princeton landmarks in mathematics and physics.

[24] R. Aumann and M. Maschler, Repeated Games with Incomplete Information. MIT Press, Cambrige, MA, 1995. 